\title{
THE IMPACT OF CLOUD COMPUTING ON IS/IT ACADEMICS
}

\author{
John E. Anderson, Utah Valley University, andersjo@uvsc.edu \\ Floyd A. Wiles, Utah Valley University, wilkesfl@uvsc.edu \\ Kevin P. Young, Utah Valley University, youngke@uvsc.edu
}

\begin{abstract}
Change in the underlying technology supporting the subjects of Information Systems and Information Technology is not new. Since the beginning of computing technology as a subject of academic interest, changing technology has been the one constant that has driven the need to adjust the content of what is taught and how it is taught. One of the most recent changes coming on the scene is the emergence of Cloud Computing. With its roots in the Google architecture and reliance on such technologies as virtualization and commodity level computers, a new computing platform is emerging that will potentially affect everything in the curriculum. This article explores some of those changes.
\end{abstract}

Keywords: cloud computing, virtualization, information systems education, information technology education

\section{INTRODUCTION}

\section{What is Cloud Computing?}

Cloud computing has emerged on the computing scene almost unnoticed by many observers. On-line giants such as Google, Yahoo, Amazon, eBay and others have been building large computer centers with enormous amounts of hardware, much of it in the form of thousands of networked commodity class computers, storing petabytes of data, and able to perform billions of queries per second to satisfy the public's appetite for search results. Now these same companies, in an effort to leverage their investment in these computer centers are offering new services designed to provide not just search capability, but to meet all of the computing needs of their customers, and they offer these services at extremely low prices and with very high levels of flexibility. They call it the Cloud and it promises to bring major changes to the way computing is done. [1] The emergence of Cloud Computing raises some interesting questions for IS and IT educators around the questions of if, how and when the existence of the cloud should change what we do as educators.
The professional literature surrounding Cloud Computing is exploding, as are the beginnings of conferences. The Hadoop Summit hosted by Yahoo which took place during the final week of March 2008 drew over 500 attendees, requiring it to change venues to accommodate the crowd. [16]

\section{Evolution of Cloud Computing}

Cloud Computing is an evolution and combination of several ideas - on-demand computing, utility computing, grid computing, software as-a-service, and thin clients - where the locus of data storage and computation shift to ever-bigger clusters of centrally managed computers. [1] A part of the Cloud Computing concept is a form of centralized timesharing, an idea bubbling up again from the dawn of the computer age. [8] Cloud computing is the computer architecture Google uses for its search service. "The next generation of computer architecture" [3]

The cloud providers seem to evolve from first renting basic data storage and then adding services like computing and databases. [8] The larger objective is thus to "support both the Internet-facing Web tier that needs to scale out to millions of users, as well as the back-end data-processing tasks" often associated with grid computing. [2]

\section{Why Consider the Cloud?}

There are some interesting arguments favoring the use of the Cloud (see Table 1). For example, by shifting computing needs to the cloud, a company is able to forego the need for building an extensive and expensive computing infrastructure and replacing it with services on the cloud where the costs are only incurred by usage. The cost of the computing capacity changes from a fixed to a variable cost.

The cloud provides an extremely flexible computing environment. For example, if a business using the Cloud were planning to run a promotion in which they expected their need for computing capacity suddenly to jump from the 200 servers they normally use to a need for 2,000 serves for a period of time, rather than meeting this need by purchasing 
additional hardware, they would simply notify their cloud provider of the need and the provider would meet the need by firing up additional servers to provide the capacity for the period needed. As soon as the need had passed, the cloud provider would simply reallocate the capacity to other needs.
The cloud makes available to smaller companies the vast computing resources enjoyed by their larger competitors but at a price based only on usage of these resources, not on the cost of acquisition.

\section{Table 1: Benefits of Cloud Computing to Organizations}

\begin{tabular}{l|l}
\hline - Cost & $\begin{array}{l}\text { Low Cost-per-instruction, a change from fixed cost to variable cost, and decreased } \\
\text { energy usage (green) by helping customers manage tens of thousands of machines in } \\
\text { the most efficient way. [2] Decreased upfront capital expenditures. [12] }\end{array}$ \\
\cline { 2 - 2 } $\begin{array}{l}\text { - Business Continuity } \\
\text { - Flexibility }\end{array}$ & $\begin{array}{l}\text { Increased technical and access flexibility with the ability to scale on demand, } \\
\text { facilitating the provisioning and allocation of resources as workloads fluctuate. The } \\
\text { ability to quickly test and prototype Web 2.0 applications with an enterprise } \\
\text { environment. [2] }\end{array}$ \\
\cline { 2 - 2 } & $\begin{array}{l}\text { For small organizations clouds mean opportunity "a leveling of the playing field in } \\
\text { the most data-intensive forms of computing." [1] Decreased technology management } \\
\text { challenges (12) "could help IT managers dramatically reduce the complexities and } \\
\text { costs of managing scale-out infrastructures whose demands fluctuate." [2] }\end{array}$
\end{tabular}

Cloud computing, having been adopted mostly by small businesses so far, is still in its early adopter phase. Most cloud computing providers aren't yet ready for enterprise-class delivery, stability, security guarantees, and iron-clad service level agreements. However, the platforms are maturing quickly. [5] New controls and safeguards will be needed to protect privacy, security, and reliability [7], caching, load balancing, fail-over, redundancy, back-end databases, and monitoring [15]. New computer programming techniques and development methods for clusters of processers [3] will also be needed.

\section{Who are the major players?}

There are several players in the Cloud Computing environment including: Amazon, Google [1] and IBM (Blue Cloud) [12, 11] partnership, and Microsoft [11] along with about nine smaller players [6]. EMC paid $\$ 75$ million for consumer online storage service Mozy which it plans to expand. [8]

In March 2008 HP launched its cloud platform aimed at large enterprise customers. It hopes that in a slowing economy, IT managers may be able to expand their infrastructures by shifting financing from capital spending to operating expenditures. HP and Amazon both promise 99.9 percent uptime. HP's cloud is optimized for SAP 6.0, with the hope that organizations can save time and money using the cloud SAP saving substantially on hardware costs and better versioning management. HP also has also targeted Microsoft Exchange users, and the ability for customers to bring their own apps to be hosted on the cloud. [6]

Salesforce.com introduced (Jan 2008) DevForce as a cloud computing architecture to allow developers to create applications without the need for software and hardware infrastructures. It feature set includes database, workflow, and Web services. Salesforce.com is also introducing utility computing capabilities to its customers that would be charged on a per-log-in basis rather than a per-seat basis which would be beneficial for applications that are used intensively at only certain times a year. This benefit would enable customers to create apps that are lower on the priority list because the utility pricing makes using the apps less expensive than traditional software models. The cloud computing environment provides resources in terms of time and money. [9]

\section{IMPACT OF CLOUD COMPUTING ON THE ACADEMIC COMMUNITY}

\section{Computer Science}

Concern for how Cloud Computing will or should affect academic programs is also appearing in the popular literature. For example, Bulkeley expresses a concern that computer science programs focus on teaching students to program a single server rather than parallel programming. [3] In January 2008 Google and IBM joined together to promote Cloud Computing research, and committed $\$ 30$ million over the next 2 years to support projects at Carnegie 
Mellon University, MIT, Stanford, University of California Berkeley, University of Maryland, and the University of Washington. The center will run a combination of Google and IBM software. [10]

A course is being offered by Thorsten von Eicken at the University of California Santa Barbara, Scalable Internet Services, focusing on the scalability and fault-tolerant features of cloud computing by having students complete a number of hands-on projects using Amazon's Simple Storage Service (S3) and Elastic Compute Cloud (EC2) services. [15]

\section{Information Systems and Information Technology}

Cloud computing will impact the IS/IT academic community at a least three levels: the department, the faculty, and the students (see Table 2).

Table 2: Impact of Cloud Computing on IS/IT

\begin{tabular}{l|c|c|c} 
& Dept Level & Faculty Level & Student Level \\
\hline Reduced Cost & High & & \\
\hline Increased Continuity & & Medium & High \\
\hline Increased Flexibility & & High & Medium \\
\hline Increased Opportunity & & High & High
\end{tabular}

\section{IS/IT Dept}

At the department level, the impact of cloud computing will be primarily financial. Reduced requirements in investment in infrastructure will free up funds to be used for software and other needs rather than an increased number of servers, increasing availability of services for the same cost.

An illustration may help to understand the costs. We will assume you are going to run a class in which students will be assigned to develop an application to run on a server. During the class we want each student to have their own virtual machine on which they will develop and test the application. We will assume that an Amazon EC2 "small" instance would be adequate for one student. An Amazon EC2 "small" instance provides: 1.7GB of memory, a single processor (i.e. 1EC2 Compute Unit), and $160 \mathrm{~GB}$ of storage on a 32-bit platform running in a Linux environment. The CPU capacity is that of a 1.0-1.2 GHz 2007 Opteron or 2007 Xeon processor. The cost of a small unit is $\$ 0.10 / \mathrm{CPU}$ hour. A CPU hour is the time the CPU is actually running. For purposes of this illustration, we will assume a class size of 20 students where each student accumulates an average of $4 \mathrm{CPU}$ hours per week. Under these assumptions it would cost $\$ 6.00$ per student per semester to provide the lab facility or $\$ 120.00$ for the class (4 CPU hrs. per week x 15 weeks x $\$ 0.10$ per hour $\mathrm{x} 20$ students). If a small instance is not sufficient, Amazon also has large instances (7.5 GB of memory; 4 EC2 Compute Units, i.e. 2 virtual cores with 2 EC2 Compute Units each; 850 GB of instance storage; 64-bit platform) at $\$ 0.40 /$ hour and extra large instances (15 GB of memory; 8 EC2 Compute Units i.e. 4 virtual cores with 2 EC2 Compute Units each; 1690 GB of instance storage; 64-bit platform) at $\$ 0.80$ /hour.

\section{Faculty}

Cloud computing will impact the faculty by making the curriculum more agile. Since the current fixed cost is changed to a variable cost based on use it is easy to add students to a class that requires server services, or even add whole classes essentially onthe-fly. This will reduce the worry and frustration faculty often face when teaching classes with infrastructure intensive hands-on components such as database and web development.

\section{Students}

At the student level, the impact of cloud computing will be a continuation of the current trends in electronic communication technology: freeing students from the tethers of time and location. Every student with access to a cloud version of a database or web server will have the luxury of 24/7 availability or resources from any location with web access. 
Another benefit of cloud computing for the student is the increased opportunity to provide instructional environments which would be impossible (or extremely expensive) otherwise. In a web server administration class, for example, one or a few servers had to be shared by teams of students. With cloud computing each student can have his/her own server instance.

\section{CONCLUSION}

The underpinnings of Cloud Computing have been evolving for some time but its emergence as a phenomenon is fairly recent. For IS and IT departments, the advent of cloud computing presents yet another requirement for change as decisions are made concerning what will be taught about cloud computing and how cloud computing may become another resource available to support teaching.

\section{Some Challenging Questions}

1. Should CS/IS/IT students be exposed to virtualization technologies?

2. Should CS/IS/IT students be exposed to the cloud provider development tools?

3. Should CS/IS/IT students be exposed to the management issues surrounding cloud computing?

\section{REFERENCES}

1. Baker, S. (2007, Dec 24) Google and the wisdom of clouds, Business Week, cover story

2. Brodkin, J. (2007, Nov 19) IBM unveils 'cloud computing:' technologies for Internet-scale computing on the way in spring 2008, Network World, Nov 19, 2007, p10

3. Bulkeley, W. (2007, Oct 8) IBM, Google, Universities combine 'cloud' forces, Wall Street Journal, New York, NY, Oct 8, 2007, pB7

4. Darrow, B. (2008, Jan 10) Amazon, eBay, Google pitch woo to developers. Redmondmag.com, retrieved Jan 14, 2008

5. Dignan, L. (2008, March 10) Cloud computing hasn't gone Fortune 500 yet, but it's coming. Between the Lines, http://blogs.zdnet.com/BTL/?p=8199\&tag=nl.e6 22, retrieved March 11, 2008

6. Dignan, L. (2008, March 17) HP launches data center as a service; the cloud meets outsourcing. Between the Lines, http://blogs.zdnet.com/BTL/?p=8231\&tag=nl.e6 22, retrieved March 17, 2008

7. Financial Times,(2008, Jan 4) A cloudy future for computing: Centralized processing will come
- but privacy is a concern, London, Jan 4, 2008, p6

8. Hardy, Q. (2008, Feb 11) The death of hardware. Forbes (181)3, p36

9. Hickins, M. (2008, Jan 21) Cloud computing gets down to earth, eWeek, Jan 21, 2008, p14

10. Lohr, S. (2007, Oct 8) Google and IBM join in 'cloud computing'research. The New York Times

11. Markoff, J. (2007, Sept 2) Software via the Internet: Microsoft in "Cloud" Computing. The New York Times

12. Papadopoulos, G. (2007, Nov 21) My advice is, if you can't take it out, don't put it in, Financial Times, London, Nov 21, 2007, p2

13. Ricadela, A. (2007, Nov 19) Computing heads for the clouds. Business Week, p28

14. Savvas, A. (2007, Nov 20) Untitled. Computer Weekly, retrieved Feb 8, 2008, from Business Source Premier database.

15. Von Eiken, T., (2006) Syllibus and class materials for CS290 at UCSB, http://www.voneicken.com/courses/ucsb-cs290ffa06/index.php/Home

16. Waters, J. (2008, March 26) Hadoop Summit: Yahoo gathers the stuffed elephant crowd. Redmond Channel Partner, RCPmag.com 